\title{
Usefulness of the platelet-to-lymphocyte ratio in predicting long-term cardiovascular mortality in patients with peripheral arterial occlusive disease
}

\author{
Fatih Uzun ${ }^{1}$, Mehmet Erturk ${ }^{1}$, Huseyin Altug Cakmak², Ali Kemal Kalkan¹, Ibrahim Faruk Akturk ${ }^{1}$, \\ Ahmet Arif Yalcin ${ }^{1}$, Begum Uygur ${ }^{1}$, Umit Bulut ${ }^{1}$, Kursat $\mathrm{Oz}^{3}$ \\ ${ }^{1}$ Department of Cardiology, Mehmet Akif Ersoy Thoracic and Cardiovascular Surgery Education and Training Hospital, Istanbul, Turkey \\ ${ }^{2}$ Department of Cardiology, Mustafakemalpasa State Hospital, Bursa, Turkey \\ ${ }^{3}$ Department of Cardiovascular Surgery, Mehmet Akif Ersoy Thoracic and Cardiovascular Surgery Education and Training Hospital, Istanbul, \\ Turkey
}

Adv Interv Cardiol 2017; 13, 1 (47): 32-38

DOI: https://doi.org/10.5114/aic.2017.66184

\begin{abstract}
A bstract
Introduction: Inflammation and increased platelet activation play a crucial role in the initiation and progression of atherosclerosis. Platelet-to-lymphocyte ratio (PLR) has recently been reported as a new independent predictor for major adverse cardiovascular events in cardiovascular diseases.

Aim: To investigate the relation between PLR and cardiovascular mortality in patients with intermittent claudication or critical limb ischemia (CLI) or both.

Material and methods: In our retrospective study, 602 consecutive patients who were admitted to a large tertiary hospital with the diagnosis of symptomatic peripheral arterial occlusive disease (PAOD) were included. Patients were divided into two groups according to their PLR as follows: high PLR (PLR > 142) and low PLR (PLR $\leq 142)$ groups.

Results: During the follow-up period (median: 33.8 months (interquartile range: 21-45)), 131 deaths occurred out of 602 (21.8\%) patients. Cardiovascular mortality was found to be significantly higher in the high PLR group compared to the low PLR group ( $31.6 \%$ vs. $17.2 \% ; p<0.001$ ). Even after adjustment for various risk factors, PLR > 142 and age were found to be independent predictors of long-term cardiovascular mortality in Cox regression analysis (hazard ratios (95\% confidence interval): 1.03 (1.01-1.04) and 1.04 (1.02-1.06), $p<0.001$ and $p<0.001$, respectively).

Conclusions: Platelet-to-lymphocyte ratio, which is one of the parameters of routine complete blood count, reflects increased inflammatory status, platelet activation and aggregation. PLR is a cheap and readily available marker that has the ability to improve risk stratification provided by conventional risk scores in predicting long-term cardiovascular mortality in PAOD.
\end{abstract}

Key words: platelet-to-lymphocyte ratio, peripheral arterial occlusive disease, cardiovascular mortality.

\section{Introduction}

Peripheral arterial occlusive disease (PAOD) is associated with high mortality and morbidity rates, especially in the elderly population. Due to the high economic, social and health burden of PAOD, early and accurate diagnosis is required for both general and high-risk populations [1]. The late diagnosis of PAOD may lead to rapid progression and development of critical limb ischemia, which is an entity with high mortality and limb amputation risk. Although modern treatment options and strategies for PAOD have improved in recent years, the rates of cardiovascular mortality and morbidity such as limb amputation still remain high [2].

Inflammation and increased platelet activation play a crucial role in the initiation and the progression of the complex atherosclerotic process that is the main underlying pathophysiologic mechanism of PAOD [3]. The pathophysiological role of inflammation in cardiovascular diseases (CVD) has been investigated with some recent studies, and a significant relation between various inflammatory markers and CVD has been reported [4, 5]. Severe chronic inflammation leads to increased proliferation in megakaryocytic series and relative thrombocytosis. Recent stud-

\section{Corresponding author:}

Fatih Uzun MD, Department of Cardiology, Mehmet Akif Ersoy Thoracic and Cardiovascular Surgery Training and Research Hospital, Istanbul, Turkey, phone: +90 5057705766, e-mail: fatihuzun28@gmail.com

Received: 6.08.2016, accepted: 24.10.2016. 
ies reported an important relation between high circulating platelet count and major adverse cardiovascular events in patients with or without known coronary artery disease history $[6,7]$. Lymphocyte count decreases due to severe apoptosis in cases of chronic severe sustained inflammation. Moreover, a decreased lymphocyte count has been reported to be associated with major adverse cardiovascular outcomes in patients with coronary artery disease and chronic heart failure $[8,9]$. The platelet-lymphocyte ratio (PLR), which is defined as the ratio of the platelet to lymphocyte count obtained from the same blood sample, has recently been demonstrated as a new inflammatory marker and significant independent predictor of major adverse cardiovascular events in various cardiovascular diseases including acute myocardial infarction. A high PLR was also demonstrated to be associated with poor prognosis in various cancers [10,11]. Gary et al. reported that high PLR was significantly related to high risk for critical limb ischemia and other cardiovascular endpoints in PAOD [12]. In addition, they demonstrated a significant correlation between PLR and some inflammatory markers such as C-reactive protein and fibrinogen.

\section{Aim}

Since there is no information in the literature concerning the relationship between PLR and long-term cardiovascular mortality both in patients with intermittent claudication and those with critical limb ischemia, we aimed to investigate this relation in the present study.

\section{Material and methods}

\section{Study design and patient selection}

Six hundred two consecutive patients, who were admitted to inpatient clinic of the vascular department of a large tertiary training and research hospital with diagnosis of symptomatic PAOD between May 2009 and September 2013, were included in this retrospective study. The PAOD was defined as $>50 \%$ stenosis in the symptomatic lower limb, which was assessed by clinical evaluation and confirmed by lower limb angiography performed according to current state-of-the-art protocols and guidelines. Symptomatic PAOD was also defined as intermittent claudication or critical limb ischemia, which included rest pain, ulceration and gangrene [13]. The categorization of PAOD was done according to the Fontaine classification. Patients were divided into two groups according to their PLR as follows: high PLR (PLR > 142) and low PLR (PLR $\leq 142)$ groups.

The exclusion criteria of the present study were as follows: patients with a history of surgical lower limb amputation as a consequence of PAOD, previous surgical or endovascular lower limb revascularization, acute infections unrelated to PAOD, recent ( $<3$ months) coronary or peripheral revascularization, recent ( $<3$ months) acute coronary syndrome or stroke, decompensated heart fail- ure, malignancy, hepatic disease, history of autoimmune disease, chronic inflammatory disease and leukocyte count above the reference limit $\left(\geq 12 \times 10^{9} / \mathrm{l}\right)$.

Demographic information including cardiovascular risk factors, comorbidities, physical examination and interventional (vascular surgery, angioplasty, stenting) data were recorded by systematic review of patients' hospital files. Also, the results of complete series of routine laboratory investigations including complete blood cell count and levels of $12 \mathrm{~h}$ overnight fasting low-density lipoprotein (LDL), high-density lipoprotein (HDL), total cholesterol and triglyceride were recorded. On admission, venous blood samples were obtained from all the patients and white blood cells count (WBC), platelets, and lymphocyte counts were measured as part of the automated complete blood count using a Coulter LH 780 Hematology Analyzer (Beckman Coulter Ireland INC, Mervue, Galway, Ireland). The PLR was calculated as the ratio of the platelets to lymphocytes, both obtained from the same automated blood sample at admission to the present study.

Firstly, the study population was divided into three groups based on their admission PLR in order to determine the association of PLR with mortality and morbidity in patients with PAOD as follows: high (PLR > 142), intermediate (PLR (95-142) and low (PLR < 95) (Figure 1). Because of the non-significant relation between intermediate and low PLR groups in terms of cardiovascular mortality in a Kaplan-Meier survival plot, the intermediate and low PLR groups were combined. The final patient

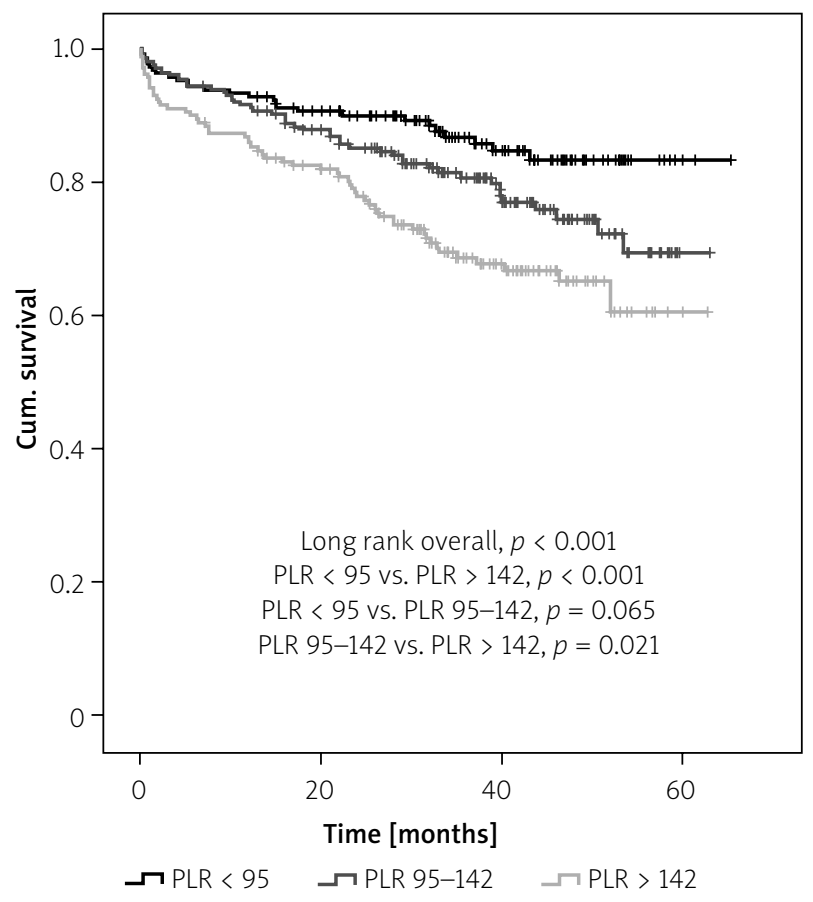

Figure 1. Kaplan-Meier curve for long-term survival according to platelet-to-lymphocyte ratio (PLR) groups. Cumulative event-free survival was defined as freedom from death 
categories were the high PLR group $(P L R>142)$ and low PLR group (NLR $\leq 142)$.

\section{Definitions}

Hypertension was diagnosed if systolic arterial pressure exceeded $140 \mathrm{~mm} \mathrm{Hg}$ and/or diastolic arterial pressure exceeded $90 \mathrm{~mm} \mathrm{Hg}$, or if the patient used antihypertensive drugs [14]. Diabetes mellitus was defined as a previous history of the disease, use of diet, insulin or oral antidiabetic drugs, or a fasting venous blood glucose level $\geq 126 \mathrm{mg} / \mathrm{dl}$ on 2 occasions in previously untreated patients [15]. Hyperlipidemia was defined as fasting total serum cholesterol > $200 \mathrm{mg} / \mathrm{dl}$, LDL cholesterol > $130 \mathrm{mg} / \mathrm{dl}$, or serum triglycerides $>180 \mathrm{mg} / \mathrm{dl}$ or if the patient used lipid-lowering drugs because of a history of hypercholesterolemia [16]. Glomerular filtration rate (GFR) for each patient was calculated using the measured plasma creatinine levels and the Cockcroft-Gault formula for the estimation of renal function [17]. The height and weight of the study participants were measured, and body mass index (BMI) was calculated as body weight in kilograms divided by the square of the height in meters $\left(\mathrm{kg} / \mathrm{m}^{2}\right)$. Smokers included current and former smokers.

\section{Study endpoints}

Follow-up data of the patients were obtained from hospital records or by telephone interview with patients, their relatives or their family physicians. The main clinical end point of the present study was cardiovascular mortality. Cardiovascular death was defined as unexplained sudden death, death due to myocardial infarction (MI), decompensated heart failure or hemodynamically significant arrhythmia.

Written informed consent was obtained from all the participants and the study was approved by the local ethics committee and institutional review board. The study protocol complied with the Declaration of Helsinki.

\section{Statistical analysis}

Continuous, normally distributed variables were expressed as mean \pm SD and non-normally distributed variables as median (interquartile range). Categorical variables were expressed as frequencies and/or percentages. The Kolmogorov-Smirnov test was used to evaluate whether the continuous variables were normally distributed. Student's $t$-test was used for the comparison of normally distributed continuous numerical variables, the Mann-Whitney $U$-test was used for non-normally distributed numerical variables, and the $\chi^{2}$ test was used for comparing categorical variables between the two groups. Any correlation between data was tested by Spearman or Pearson correlation analysis. Univariate and backward stepwise multivariate Cox regression analyses, which included variables with a $p$-value less than 0.1 , were performed to identify independent predictors of cardiovascular mortality. Survival analysis was conducted using Kaplan-Meier survival curves, and differences were compared using the log-rank test. A two-sided $p$-value was considered for all comparisons. Statistical significance was defined as $p<0.05$. Statistical analyses were carried out using the Statistical Package for Social Sciences for Windows 17.0 (SPSS Inc., Chicago, Illinois, USA).

\section{Results}

We recruited 713 patients who presented with symptomatic PAOD during the study period. One hundred and eleven (15.5\%) patients had to be excluded due to missing data about platelet and/or lymphocyte counts or follow-up results. Therefore, the final study population consisted of 602 (median age: 64 years; IQR: 56-70, 78.7\% male) patients. The composition of the study groups was as follows: 190 patients in the high PLR group (NLR $>142$ ) and 412 patients in low PLR group (PLR $\leq 142)$. Baseline demographic and clinical characteristics of the patients according to PLR groups are presented in Table I. The high PLR group was significantly older $(p<0.001)$ and had a higher frequency of critical limb ischemia and renal failure ( $p<0.001$ and $p=0.001$ respectively) than the low PLR group. However, the incidence of intermittent claudication in the low PLR group was higher than the high PLR group $(p<0.001)$. There was no difference between two groups in terms of other demographic and clinical characteristics (Table I). Furthermore, the distribution of patients according to severity of critical limb ischemia (CLI) was as follows: rest pain (Fontaine stage 3, Rutherford stage 4) 59\%; ischemic ulceration (Fontaine stage 4, Rutherford stage 5) 31.8\%; gangrene (Fontaine stage 4, Rutherford stage 6) $9 \%$.

\section{White blood cells counts}

The median total leukocyte count was $8.6 \times 10^{9} / 1$ (IQR: 7.3-9.9), consisting of median counts of $5.2 \times 10^{9} / \mathrm{l}$ for neutrophils (IQR: 4.4-6.4), $2.2 \times 10^{9} /$ for lymphocytes (IQR: 1.6-2.7), $257 \times 10^{3} / \mathrm{l}$ for platelets (IQR: 208-304). There was no difference between two groups in terms of WBC count ((high PLR group; median: 8.1 (IQR: 6.7-10.2) vs. (low PLR group; median: 8.7 (IQR: 7.5-9.8) × 109/l, $p=0.168)$ ). Baseline neutrophil and platelet values were higher in the high PLR group (median: 5.7 (IQR: 4.6-7.4) vs. median: 5.1 (IQR: 4.3-96.1) × 109/l, $p<0.001$; median: 304 (IQR: 244-382) vs. median: 236 (IQR: 198-281) $\times 10^{3} /$ l, $p<0.001$, respectively) than the low PLR group. However, baseline lymphocyte values were lower in the high PLR group (median: 1.6 (IQR: 1.2-1.9) vs. median: 2.4 (IQR: 2.0-3.0) × 10\%/, $<<0.001$ ). The PLR showed a poor but significant correlation with the Fontaine stage of PAOD $(r=0.167, p<0.001)$.

During the course of the present study (median follow-up period of 33.8 months (IQR: 21-45)), 131 deaths 
Table I. Baseline demographic and clinical characteristics of the study groups according to PLR

\begin{tabular}{|c|c|c|c|}
\hline Variable & PLR $\leq 142(n=412)$ & PLR $>142(n=190)$ & $P$-value \\
\hline Age, median (IQR) [years] & $62(55-69)$ & $68(55-73)$ & 0.001 \\
\hline Body mass index, median (IQR) $\left[\mathrm{kg} / \mathrm{m}^{2}\right]$ & $27.4(25.0-29.7)$ & $27.4(23.9-30.1)$ & 0.603 \\
\hline Male gender, $n(\%)$ & $332(80.6)$ & $142(74.7)$ & 0.103 \\
\hline Diabetes mellitus, $n(\%)$ & $167(41.6)$ & $81(43.5)$ & 0.664 \\
\hline Hypertension, $n(\%)$ & $232(61.2)$ & $123(67.2)$ & 0.167 \\
\hline Hypercholesterolemia, $n$ (\%) & $233(62.3)$ & $102(55.7)$ & 0.137 \\
\hline Smokers, $n(\%)$ & $219(56.6)$ & $108(58.7)$ & 0.634 \\
\hline Renal failure, $n(\%)$ & $68(16.6)$ & $53(28.2)$ & 0.001 \\
\hline Coronary artery disease, $n$ (\%) & $203(49.3)$ & $89(46.8)$ & 0.579 \\
\hline Previous myocardial infarction, $n$ (\%) & $76(18.4)$ & $38(20)$ & 0.651 \\
\hline Previous stroke, $n$ (\%) & $12(2.9)$ & $9(4.7)$ & 0.257 \\
\hline \multicolumn{4}{|l|}{ Symptoms, $n$ (\%): } \\
\hline Intermittent claudication & $354(85.9)$ & $138(72.6)$ & \multirow[t]{2}{*}{0.001} \\
\hline Critical limb ischemia & $58(14.1)$ & $52(27.4)$ & \\
\hline \multicolumn{4}{|l|}{ Peripheral procedure, $n$ (\%): } \\
\hline Vascular surgery & $60(14.6)$ & $41(21.6)$ & \multirow{3}{*}{0.096} \\
\hline Angioplasty/stenting & $147(35.7)$ & $60(31.6)$ & \\
\hline Conservative/palliative & $205(49.8)$ & $89(46.8)$ & \\
\hline \multicolumn{4}{|l|}{ Medications, $n(\%)$ : } \\
\hline Acetylsalicylic acid & $313(79.2)$ & $150(81.1)$ & 0.607 \\
\hline Clopidogrel & $130(33.6)$ & $60(32.4)$ & 0.783 \\
\hline Statin & $145(39.1)$ & $64(35.4)$ & 0.397 \\
\hline
\end{tabular}

$P L R$ - platelet-to-lymphocyte ratio.

occurred out of 602 (21.8\%) patients. The distribution of causes of cardiovascular mortality were as follows: 67 patients with acute myocardial infarction, 36 patients with decompensated heart failure, 12 patients with unexplained sudden death and 16 patients with hemodynamically significant arrhythmia. Cardiovascular mortality was found to be significantly higher in the high PLR group $(n=60)$ compared to the low PLR group $(n=71)$ (31.6\% vs. $17.2 \% ; p<0.001)$. The Kaplan-Meier survival plot for cardiovascular mortality in both groups is presented in Figure 2.

The independent predictors for cardiovascular mortality including age, gender, hypertension, smoking status, previous stroke history, hyperlipidemia, diabetes mellitus, coronary artery disease, renal failure, Fontaine category, preprocedural PLR tertiles and use of statin were included in a Cox regression model and analyzed in a stepwise fashion. The PLR > 142 and age were found to be independent predictors of cardiovascular mortality after adjustment for other risk factors such as renal fail- ure, hypertension and Fontaine category, which had been found to be significant predictors of mortality in unadjusted analysis (hazard ratios (95\% confidence interval): 1.03 (1.01-1.04) and 1.04 (1.02-1.06), $p<0.001$ and $p<$ 0.001 respectively; Table II).

\section{Discussion}

The main findings of the present study were: (1) patients in the high PLR group were older and had higher prevalence of critical limb ischemia than the low PLR group; (2) PLR showed a poor but significant correlation with the severity of PAOD defined as Fontaine stage; (3) age and high PLR were demonstrated to be independent predictors of long-term cardiovascular mortality after adjustment for other risk factors in patients with PAOD.

The PLR, which is defined as the ratio of the platelet to lymphocyte count, integrates the risk predictor effect of two parameters into a single risk factor. It reflects increased inflammation, platelet activation and aggregation. Hence, PLR may be acceptable as a more valuable 


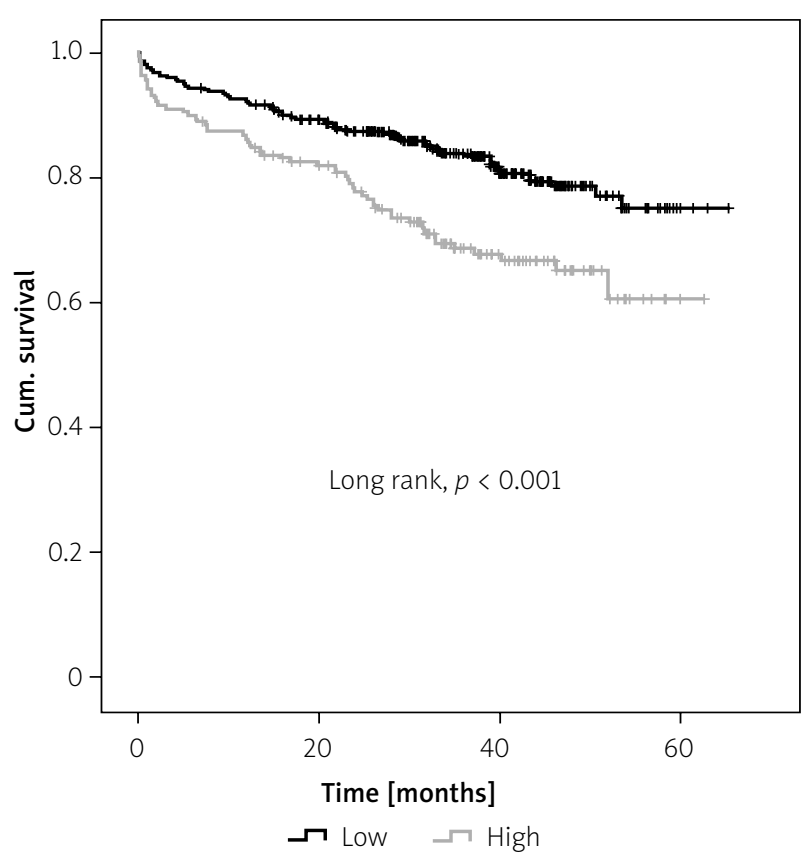

Figure 2. Kaplan-Meier curve for long-term survival according to platelet-to-lymphocyte ratio (PLR) with a cutoff of 142.0. Cumulative event-free survival was defined as freedom from death

indicator than either platelet or lymphocyte count alone in the prediction of peripheral atherosclerotic burden. Previous clinical studies demonstrated a significant relation of increased PLR and major adverse outcomes in various cardiovascular diseases [18, 19]. It was also reported that raised PLR was associated with severity and complexity of coronary artery disease [20], coronary slow flow [21], no-reflow after primary percutaneous coronary intervention [22, 23], poor coronary collateral development [24, 25], and non-dipper hypertension [26]. Moreover, Azab et al. demonstrated high PLR as a significant independent predictor of long-term mortality in non-ST segment elevation myocardial infarction [27]. A strong positive relation between increased PLR and in-hospital mortality was also reported by Temiz et al. [28]. Gary et al. demonstrated a significant association of high PLR with high risk for critical limb ischemia and other cardiovascular end points in patients with PAOD [29]. They were able to report that PLR > 150 can be used to discriminate patients at high risk for $\mathrm{CLI}$ from those with a low CLI risk. The predictive role of PLR for limb salvage in patients with CLI was also confirmed by the study of Tașoğlu et al. [30]. The CLI patients with high risk for limb amputation during long-term follow-up were differentiated from patients with lower risk for limb amputation by PLR.

To the best of our knowledge, this is the first study which has demonstrated a significant independent association between high PLR and long-term cardiovascular mortality in both patients presenting with intermittent claudication and those with critical limb ischemia. The possible theory for this finding may be that increased blood viscosity due to higher platelet count leads to higher PLR. Severe inflammation, which is seen in atherosclerosis such as PAOD, increases platelet activation and aggregation and results in a higher platelet count. A low lymphocyte count was also detected in chronic severe inflammatory states due to severe apoptosis. More-

Table II. Cox regression analysis for predictors of long-term cardiovascular mortality

\begin{tabular}{|c|c|c|c|c|}
\hline \multirow[t]{2}{*}{ Parameter } & \multicolumn{2}{|c|}{ Unadjusted } & \multicolumn{2}{|c|}{ Adjusted } \\
\hline & Odds ratio $(95 \% \mathrm{Cl})$ & $P$-value & Odds ratio $(95 \% \mathrm{Cl})$ & $P$-value \\
\hline Age & 1.06 (1.04-1.08) & 0.001 & $1.04(1.02-1.06)$ & 0.001 \\
\hline Sex & $0.91(0.61-1.38)$ & 0.673 & & \\
\hline Hypertension & $1.37(0.94-1.96)$ & 0.098 & $1.25(0.85-1.82)$ & 0.258 \\
\hline Smoking status & $1.00(0.71-1.43)$ & 0.987 & & \\
\hline Previous stroke & $0.23(0.03-15.6)$ & 0.129 & & \\
\hline Hypercholesterolemia & $0.79(0.56-1.11)$ & 0.174 & & \\
\hline Diabetes mellitus & $1.17(0.83-1.66)$ & 0.375 & & \\
\hline Coronary artery disease & $0.83(0.57-1.17)$ & 0.296 & & \\
\hline Renal failure & $2.07(1.44-2.98)$ & 0.001 & $1.43(0.97-2.12)$ & 0.070 \\
\hline Fontaine category & $1.79(1.22-2.62)$ & 0.003 & $1.46(0.97-2.18)$ & 0.067 \\
\hline $\mathrm{PLR}>142$ & $1.04(1.03-1.05)$ & 0.001 & $1.03(1.01-1.04)$ & 0.001 \\
\hline Statin use & $1.10(0.77-1.57)$ & 0.615 & & \\
\hline
\end{tabular}

$P L R$ - platelet-to-lymphocyte ratio. 
over, a poor but significant relation was demonstrated between higher PLR and severity of PAOD defined as Fontaine stage. Higher PLR was also presented to be correlated with other inflammatory markers such as C-reactive protein (CRP) concordant with the study of Gary et al. These findings support an important link between increased inflammation, triggered platelet activity and aggregation, lymphocyte suppression by apoptosis and severity of atherosclerosis such as PAOD.

Platelets play a pivotal role in the initiation and the progression of atherosclerosis by interacting with endothelial cells and leukocytes and releasing inflammatory mediators, cytokines, which leads to increased adhesion and transmigration of monocytes, and may support inflammation in the vessel wall. Severe inflammation stimulates megakaryocytic proliferation and produces relative thrombocytosis. It may be speculated that higher platelet count, which is a marker of a prothrombotic state, may represent higher propensity to form platelet-rich arterial thrombi on atherosclerotic plaques in the coronary and/ or peripheral arterial tree, leading to major adverse cardiovascular events such as mortality. Moreover, not only platelets but also microparticles derived from platelets have procoagulant activity enabling platelets take a central role in hemostasis and thrombosis. They were also reported to be related to formation and progression of arterial thrombi following injury of the vessel structure, leading to impaired functions [31]. High PLR might enhance microparticle production, triggering hemostasis and resulting in increased vascular end points such as $\mathrm{CLI}$ and long-term cardiovascular mortality. Lymphocyte count, which is the second constituent of the PLR, plays an important role in the course of atherosclerosis. High lymphocyte count was demonstrated in the limb salvage group by the study of Iso et al. [32]. The possible mechanism of this finding may be the mediation of collateral vessel growth via interleukin-16 by lymphocytes. This might also be an explanation for our findings. Patients with a high lymphocyte count, which leads to lower PLR, might have more collateral vessel growth, resulting in less ischemia and hence less CLI and long-term cardiovascular mortality.

The high PLR group was significantly older than the patients in the low PLR group, concordant with another recent study conducted in this setting [30]. Age was found to be a significant independent predictor of longterm cardiovascular mortality in patients with PAOD. Furthermore, a relation between high PLR and long-term cardiovascular mortality remained significant even after adjustment for age in our study.

The superiority and advantages of PLR to either individual lymphocyte or platelet count can be explained by two factors. The first is the stability of PLR compared to the absolute platelet or lymphocyte count, which could be altered by several physiological and pathological conditions. Second, PLR represents two inversely associ- ated predictors which play roles in atherosclerosis and immune pathways. We hypothesize that high PLR is an important marker of an ongoing destructive inflammatory response and the pro-thrombotic state, which are two crucial pathophysiological mechanisms for PAOD.

\section{Study limitations}

There are some limitations in the present study. First, this study had a retrospective design and arose from a single center, which can lead to selection bias. Second, because of single measurement of PLR on admission, the changes in response to treatment cannot be evaluated. Third, the end point of the study was limited only with cardiovascular mortality. The lack of measurements of well-known atherosclerosis indicators such as ankle brachial index, ultrasonographic and angiographic indexes of the extent of PAOD and inflammatory markers such as CRP in patients with PAOD was the last limitation of this study.

\section{Conclusions}

We demonstrated that high PLR was associated with higher long-term cardiovascular mortality in patients with PAOD, who were admitted for critical limb ischemia or intermittent claudication. Age and PLR > 142 were found to be independent significant predictors for longterm cardiovascular mortality in PAOD even after adjustment for various risk factors. The PLR, which is one of the parameters of routine complete blood count and reflects increased inflammatory status and platelet activation and aggregation, is a cheap and readily available marker that has an ability to improve risk stratification provided by conventional risk scores in predicting long-term cardiovascular mortality in PAOD.

\section{Conflict of interest}

The authors declare no conflict of interest.

\section{References}

1. Fowkes FG, Rudan D, Rudan I, et al. Comparison of global estimates of prevalence and risk factors for peripheral artery disease in 2000 and 2010: a systematic review and analysis. Lancet 2013; 382: 1329-40.

2. Novo S, Coppola G, Milio G. Critical limb ischemia: definition and natural history. Curr Drug Targets Cardiovasc Haematol Disord 2004; 4: 219-25.

3. Hansson GK. Inflammation, atherosclerosis, and coronary artery disease. N Engl J Med 2005; 352: 1685-95.

4. Han YC, Yang TH, Kim DI, et al. Neutrophil to lymphocyte ratio predicts long-term clinical outcomes in patients with ST-segment elevation myocardial infarction undergoing primary percutaneous coronary intervention. Korean Circ J 2013; 43: 93-9.

5. Erturk M, Cakmak HA, Surgit O, et al. The predictive value of elevated neutrophil to lymphocyte ratio for long-term cardiovascular mortality in peripheral arterial occlusive disease. J Cardiol 2014; 64: 371-6. 
6. Nikolsky E, Grines CL, Cox DA, et al. Impact of baseline platelet count in patients undergoing primary percutaneous coronary intervention in acute myocardial infarction (from the CADILLAC trial). Am J Cardiol 2007; 99: 1055-61.

7. Thaulow E, Erikssen J, Sandvik L, et al. Blood platelet count and function are related to total and cardiovascular death in apparently healthy men. Circulation 1991; 84: 613-7.

8. Acanfora D, Gheorghiade M, Trojano L, et al. Relative lymphocyte count: a prognostic indicator of mortality in elderly patients with congestive heart failure. Am Heart J 2001; 142: 167-73.

9. Ommen SR, Gibbons RJ, Hodge DO, et al. Usefulness of the lymphocyte concentration as a prognostic marker in coronary artery disease. Am J Cardiol 1997; 79: 812-4.

10. Kwon HC, Kim SH, Oh SY, et al. Clinical significance of preoperative neutrophil-lymphocyte versus platelet-lymphocyte ratio in patients with operable colorectal cancer. Biomarkers 2012; 17: 216-22.

11. Smith RA, Bosonnet L, Raraty M, et al. Preoperative platelet-lymphocyte ratio is an independent significant prognostic marker in resected pancreatic ductal adenocarcinoma. Am J Surg 2009; 197: 466-72.

12. Gary T, Pichler M, Belaj K, et al. Platelet-to-lymphocyte ratio: a novel marker for critical limb ischemia in peripheral arterial occlusive disease patients. PLoS One 2013; 8: e67688.

13. Rooke TW, Hirsch AT, Misra S, et al. Society for Cardiovascular Angiography and Interventions; Society of Interventional Radiology; Society for Vascular Medicine; Society for Vascular Surgery. 2011 ACCF/AHA Focused Update of the Guideline for the Management of Patients With Peripheral Artery Disease (updating the 2005 guideline): a report of the American College of Cardiology Foundation/American Heart Association Task Force on Practice Guidelines. J Am Coll Cardiol 2011; 58: 2020-45.

14. James PA, Oparil S, Carter BL, et al. 2014 evidence-based guideline for the management of high blood pressure in adults: report from the panel members appointed to the Eighth Joint National Committee (JNC 8). JAMA 2014; 311: 507-20.

15. Kadoglou NP, Iliadis F, Angelopoulou N, et al. Cardiorespiratory capacity is associated with favourable cardiovascular risk profile in patients with type 2 diabetes. J Diabetes Complications 2009; 23: $160-6$

16. European Association for Cardiovascular Prevention \& Rehabilitation, Reiner Z, Catapano AL, De Backer G, et al. ESC Committee for Practice Guidelines (CPG) 2008-2010 and 2010-2012 Committees. ESC/EAS Guidelines for the management of dyslipidaemias: the Task Force for the management of dyslipidaemias of the European Society of Cardiology (ESC) and the European Atherosclerosis Society (EAS). Eur Heart J 2011; 32: 1769-818.

17. Cockcroft DW, Gault MH. Prediction of creatinine clearance from serum creatinine. Nephron 1976; 16: 31-41.

18. Vidwan P, Lee S, Rossi JS, et al. Relation of platelet count to bleeding and vascular complications in patients undergoing coronary angiography. Am J Cardiol 2010; 105: 1219-22.

19. Zouridakis EG, Garcia-Moll X, Kaski JC. Usefulness of the blood lymphocyte count in predicting recurrent instability and death in patients with unstable angina pectoris. Am J Cardiol 2000; 86: 449-51.

20. Kurtul A, Murat SN, Yarlioglues M. Association of platelet-to-lymphocyte ratio with severity and complexity of coronary artery disease in patients with acute coronary syndromes. Am J Cardiol 2014; 114: 972-8.
21. Oylumlu $M$, Doğan A, Oylumlu $M$, et al. Relationship between platelet-to-lymphocyte ratio and coronary slow flow. Anatol J Cardiol 2015; 15: 391-5.

22. Kurtul A, Yarlioglues M, Murat SN, et al. Usefulness of the platelet-to-lymphocyte ratio in predicting angiographic reflow following primary percutaneous coronary intervention in patients with acute ST-segment elevation myocardial infarction. Am J Cardiol 2014; 114: 342-7.

23. Toprak C, Tabakci MM, Simsek Z, et al. Platelet/lymphocyte ratio was associated with impaired myocardial perfusion and both in-hospital and long-term adverse outcome in patients with ST-segment elevation acute myocardial infarction undergoing primary coronary intervention. Postep Kardiol Interw 2015; 11 : 288-97.

24. Açar G, Kalkan ME, Avci A, et al. The relation of platelet-lymphocyte ratio and coronary collateral circulation in patients with stable angina pectoris and chronic total occlusion. Clin Appl Thromb Hemost 2015; 21: 462-8.

25. Akdag S, Akyol A, Asker M, et al. The relation of platelet-lymphocyte ratio and coronary collateral circulation in patients with non-ST segment elevation myocardial infarction. Postep Kardiol Interw 2016; 12: 224-30.

26. Sunbul M, Gerin F, Durmus E, et al. Neutrophil to lymphocyte and platelet to lymphocyte ratio in patients with dipper versus non-dipper hypertension. Clin Exp Hypertens 2014; 36: 217-21.

27. Azab B, Shah N, Akerman M, et al. Value of platelet/lymphocyte ratio as a predictor of all-cause mortality after non-ST-elevation myocardial infarction. J Thromb Thrombolysis 2012; 34: 326-34.

28. Temiz A, Gazi E, Güngör Ö, et al. Platelet/lymphocyte ratio and risk of in-hospital mortality in patients with ST-elevated myocardial infarction. Med Sci Monit 2014; 20: 660-5.

29. Gary T, Pichler M, Belaj K, et al. Platelet-to-lymphocyte ratio: a novel marker for critical limb ischemia in peripheral arterial occlusive disease patients. PloS One 2013; 8: e67688.

30. Taşoğlu I, Sert D, Colak N, et al. Neutrophil-lymphocyte ratio and the platelet-lymphocyte ratio predict the limb survival in critical limb ischemia. Clin Appl Thromb Hemost 2013; 20: 645-50.

31. Suades R, Padró T, Vilahur G, et al. Circulating and platelet-derived microparticles in human blood enhance thrombosis on atherosclerotic plaques. Thromb Haemost 2012; 108: 1208-19.

32. Iso Y, Soda T, Sato T, et al. Impact of implanted bone marrow progenitor cell composition on limb salvage after cell implantation in patients with critical limb ischemia. Atherosclerosis 2004; 209: 167-72. 